\title{
Rogers BRUBAKER, Trans Gender and Race in an Age of Unsettled Identities
}

\author{
Olga L. Gonzalez
}

\section{(2) OpenEdition}

1 Journals

Édition électronique

URL : https://journals.openedition.org/ress/4074

DOI : $10.4000 /$ ress. 4074

ISSN : 1663-4446

Éditeur

Librairie Droz

\section{Édition imprimée}

Date de publication : 14 décembre 2018

Pagination : 288-293

ISSN : 0048-8046

\section{Référence électronique}

Olga L. Gonzalez, "Rogers BRUBAKER, Trans Gender and Race in an Age of Unsettled Identities », Revue européenne des sciences sociales [En ligne], 56-2 | 2018, mis en ligne le 14 décembre 2018, consulté le 06 janvier 2022. URL : http://journals.openedition.org/ress/4074 ; DOI : https://doi.org/10.4000/ress. 4074

Ce document a été généré automatiquement le 6 janvier 2022.

(c) Librairie Droz 


\title{
Rogers BRUBAKER, Trans Gender and Race in an Age of Unsettled Identities
}

\author{
Olga L. Gonzalez
}

\section{RÉFÉRENCE}

Rogers BRUBAKER, 2016, Trans Gender and Race in an Age of Unsettled Identities, Princeton University Press, 256 p.

1 Rogers Brubaker, sociologue états-unien rattaché à l'UCLA, est très connu pour ses apports dans le champ des relations interethniques. Sa critique de concepts usuels dans les sciences sociales (tel celui d'identité) et ses efforts pour employer un langage analytique plus précis a contribué à enrichir ce champ d'études.

2 Interpellé par les « affaires Dolezal et Jenner », amplement relayées aux États-Unis et ayant donné lieu à des prises de position acerbes, Brubaker s'attaque à un terrain nouveau: le genre. Rappelons les faits: en juin 2015, Rachel Dolezal, 37 ans et présidente d'une section de la National Association for the Advancement of Colored People, NAACP, qui s'était toujours présentée comme étant "noire", et alors qu'elle avait montré une grande implication pour le mouvement noir depuis son adolescence, a été outée par ses parents (chrétiens fondamentalistes) comme étant «blanche ». L'outing a entraîné sa démission de ses fonctions à la NAACP et suscita une grande vague de réprobations.

Parallèlement, en juin de cette même année, les États-Unis suivaient avec fascination la transformation de Bruce Jenner en Caitlyn Jenner. L'ancien champion olympique, icône sportive et figure médiatique (il était le patriarche dans la série très suivie The Kardashians) avait soigné son "coming out " en tant que femme trans (interview à la télévision, portrait dans Vanity Fair). Elle était devenue la personne transgenre la plus célèbre aux États-Unis (et possiblement au monde), et avait été félicitée unanimement par son courage (même le président Obama l'avait soutenue). 
4 Ce sont ces deux types de réaction, et les débats auxquels ils ont donné suite que le spécialiste des relations entre les races a analysés. D'emblée, l'auteur rappelle que les mots "transgenre " et "transracial » résonnaient auprès du public de manière différente. Le mot "transgenre ", qui dans son acception populaire se réfère au changement d'une catégorie du sexe clairement identifiable vers une autre catégorie, identifiable également, est très populaire -l'auteur note combien il se prête à une forme narrative consacrée, le tragique décalage entre le corps intimement ressenti et le corps social, attribué (par erreur) à la naissance. Le mot «transracial», lui, n'avait été employé que dans le contexte spécifique des adoptions interraciales, lesquelles sont par ailleurs très critiquées aux États-Unis. Pendant l'été 2015, on a assisté à la réapparition du mot «transracial » avec le sens de « changement de race ». Dès lors, "transgenre » et « transracial » étaient mis sur un même plan.

positions à l'égard de la possibilité de changer de sexe ou de race ont émergé selon le double registre "essentialisme/ volontarisme " et "racial/de genre ». Pour Brubaker, «l'essentialisme » est l'idée que les identités sont définies par la nature ou par l'histoire, tandis que le "volontarisme » met l'accent sur le choix, même lorsque l'on admet que l'identité originelle peut ne pas relever de l'ordre du choix (p. 22). En somme, le volontarisme permet le libre choix de la présentation de soi.

Brubaker montre que la majorité de la gauche (et des universitaires) se situait dans la case « volontarisme de genre (on peux choisir son genre) et essentialisme de "race" (on ne peut pas choisir sa race) ». Ce secteur parlait « d'appropriation culturelle » dans le cas Dolezal (qui avait osé choisir sa race) mais ne contestait pas la légitimité du cas Jenner (qui avait eu le courage de choisir son genre). Brubaker commente ce clivage de la part des secteurs les plus influents. Pour eux, le libre choix de la race aboutirait à un affaiblissement de la «race» (et de la communauté afro-américaine), là où les réclamations des trans étaient perçues comme une affaire de droits humains.

7 Sur la base de très nombreux exemples, où l'on voit que le " passing » de Dolezal est loin d'être unique, Brubaker montre que les identités de genre et de race sont devenues instables et de plus en plus contestées. La plus grande visibilité des questions trans et intersexe (y compris sur le plan légal et social, comme les passeports sans mention de sexe en Australie ou les multiples identifications de genre sur Facebook) en sont une manifestation centrale. Concernant la race, la diversification des flux migratoires, l'augmentation des mariages mixtes, la contestation des systèmes de classification raciale en sont autant d'éléments.

8 Dans ce contexte, «l'empire du choix » semble se diffuser : si les combats féministes d'hier ont permis de choisir son partenaire sexuel ou de décider sur sa grossesse (p. 51), aujourd'hui on choisit son orientation et/ou identité sexuelle. Pour la race, les cases imposées, limitées aux catégories "blanc» et "noir» sont une affaire du passé. Aujourd'hui, le mélange ethnique est devenu usuel, et l'auto-désignation la règle dans l'administration aux États-Unis. Certes, il existe aussi une «surveillance de l'identité déclarée » (p. 56). Malgré cette tendance générale vers la remise en cause des identités de genre et de race, l'auteur remarque que tandis que faiblit le pouvoir des disciplines qui hier certifiaient de l'authenticité des personnes transgenre (psychiatrie et médecine), le cas Dolezal montre que la sanction sociale à l'égard des «fraudes à la race » reste vive (p. 63).

9 Pour le sociologue qu'est Brubaker, la question est désormais de savoir sur quel fondement se construisent ces identités (ou leur sanction). Or, il observe plusieurs 
paradoxes. Le premier est qu'alors que les bases génétiques relatives à la connaissance « raciale» des aïeuls progresse (via, notamment, l' ancestry shopping, l'achat de renseignements génétiques à partir d'échantillons de son corps), cette même base biologique perd du poids en tant qu'argument pour prouver son appartenance raciale. En effet, même si la « race » inclut toujours des caractéristiques phénotypiques, elle a aussi une grande composante relationnelle (la communauté décide qui est « noir », et il ne suffit pas d'avoir un gène africain). Le deuxième paradoxe est que, alors que depuis un demi-siècle le genre et le sexe sont perçus de manière dissociée, il y a dans ce champ un retour à des revendications essentialistes. L'orientation et l'identité relèvent ici de plus en plus de l'ordre du donné (et non pas du choisi), d'une « objectivité » et non pas d'une « subjectivité ». Celui-ci est un point central : la « subjectivité » volontariste, une idée moderne, est parfois dépassée par l'« objectivité » essentialiste, que l'on croyait révolue. Le plus surprenant dans ce contexte, avance Brubaker, est que le langage essentialiste est employé non seulement par les pourfendeurs des controverses identitaires (par exemple, par les défenseurs de la morale traditionnelle) mais aussi par ses défenseurs (par exemple, les partisans des droits des personnes trans avancent souvent des arguments biologiques, alors qu'il n'y a aucune étude conclusive à ce sujet, p. 65).

10 Ce cadre, présenté dans la première partie de l'ouvrage, est suivi de la proposition contenue dans la deuxième partie consistant à penser les questions raciales et de sexe à travers le prisme de lecture "trans". Il expose trois typologies: les "trans qui migrent », les « trans de l'entre-deux » et les « trans de l'au-delà ».

11 Le premier cas de figure, « les trans qui migrent », suppose un changement définitif, on se déplace d'une catégorie vers l'autre (par exemple, homme vers femme, ou "noir » vers «blanc»). Ce type de transition est le plus couramment associé au préfixe «trans». Fréquent aujourd'hui pour le changement de sexe, il est moins bien admis pour les changements de race, à l'exception des personnes ayant « migré » vers la race amérindienne. La raison? Brubaker rappelle que tout changement de catégorie est aussi un changement de statut. Pourtant, dans l'histoire des États-Unis, il existe quelques exemples de transition de race - retenons celui du musicien de jazz d'origine grecque Johnny Otis, qui affirmait : «Si notre société allait nous dicter qu'il fallait être noir ou blanc, jeune, j'ai choisi d'être noir» (p. 88). Néanmoins, la suspicion d'appropriation culturelle plane et la communauté censure les tentatives de changement: Dolezal en a fait les frais parce qu'elle a inventé un ancêtre noir. En contraste, les rappeurs blancs qui agissent publiquement dans des modalités «nonblanches » se gardent bien de se déclarer « noirs »... Ainsi, la seule manière admise de passer pour un « noir » est de garder son identité blanche.

12 Dans la catégorie des "trans de l'entre deux", on refuse l'appartenance définitive à une seule identité. La combinaison des identités (dans le cas du genre, masculines et féminines) est ici la règle; elle se manifeste, par exemple, dans les grossesses des hommes trans. Brubaker rappelle que cette idée est ancienne (ainsi, la psychologie a parlé de tendance à la bisexualité universelle à l'aube du $\mathrm{xx}^{\mathrm{e}}$ siècle). Dans le cas de la " race ", les choses sont un peu plus complexes. L'auteur montre que les tests ADN font désormais envisager l'appartenance raciale comme une palette où la mixité est la norme. Cependant, quand il s'agit de s'auto-identifier, la «race» retenue est la plus proche (à savoir, celle de ses parents). Celle-ci est plus figée que la « race » issue des tests ADN. Ceci montre que les personnes persistent à définir des contours stricts entre 
les « races ». Parallèlement, cependant, la tendance des nouvelles générations issues de parents de "races" différentes est de prendre en compte plusieurs appartenances, revendiquant donc la mixité (p. 107). Il reste que la classification faite par des tiers sur ces personnes est encore rigide (une personne née de père «blanc» et de mère «noire" sera perçu comme "noire", quand bien même elle se définirait comme «blanche » et « noire »). Ainsi, en matière de « race », les «trans de l'entre-deux » font l'objet de plus de réticences.

13 Les «trans de l'au-delà ", de leur côté, refusent les catégories ou veulent les dépasser. Se retrouvent ici ceux qui s'opposent à la binarité des sexes, ceux qui mettent l'accent sur la performance du genre (au lieu de son énonciation), ou ceux qui se déclarent "sans genre ". Les apports conceptuels des féministes sont riches dans ce domaine elles ont réfléchi depuis des années à une société sans genre. Cependant, les conditions sociales, et notamment l'inégalité historique entre les femmes et les hommes, rendent difficile une pensée et une action politique en commun avec les trans. En ce qui concerne la «race ", Brubaker explique que la notion de "multi-racial», propre aux États-Unis, est à mettre dans ce registre (p.123). Cependant, les enjeux politiques constituent un puissant frein : ainsi, si les militants de la case «multi-raciale » dans le recensement officiel n'ont pas obtenu gain de cause, c'est en partie parce que les groupes «noirs » auraient pu voir leur nombre baisser - et, corrélativement, les fonds destinés à la population noire. Brubaker rappelle également que les opposants à l'identification des «races" le font aussi bien au nom du libéralisme (la classification par « races » a été utilisée historiquement contre la démocratie) que du radicalisme (ainsi du mouvement de Noël Ignatiev, qui prône un abandon, par les blancs, de leur « race »). En somme, en ce qui concerne la « race », l'auteur constate que l'idée d'aller vers un dépassement est très minoritaire.

On l'aura compris, en utilisant la grille «trans » comme outil analytique, Brubaker fournit un essai de synthèse richement documenté sur deux transformations sociales d'envergure, celle relative au genre et celle relative à la « race ». Il montre combien une transformation très importante, à savoir l'acceptation grandissante de l'idée que le genre n'est pas une donnée invariable et fixe, est survenue en un laps de temps très réduit. L'ouvrage illustre également la grande diversité des identités possibles chez les personnes transgenre. Les chapitres sur les "trans de l'entre-deux» (p.92), et les «trans de l'au-delà » (p. 113) contribuent à enrichir un regard au-delà de la binarité homme/femme, alors que les média mettent en avant les profils des «trans qui migrent ", de nature à renforcer cette binarité.

Le livre attire l'attention sur la situation paradoxale de ce xxI siècle : alors qu'il y a une conscience accrue de la construction sociale des « races » (on ne "devient " pas noir sur la base stricte du choix, la communauté a son mot à dire), en ce qui a trait aux questions relatives aux personnes trans, on assiste à une essentialisation (biologique et/ou psychologique) du sexe. Ici, la décision de la transition obéit à un ressort qui relève de l'intime ; le chemin est individuel, même s'il est fêté par la collectivité dans son ensemble. Que traduit cette forme très différente de conception des identités? Brubaker affirme que tout changement d'identité est aussi un changement de statut. Cependant, le traitement différencié de ces deux transitions n'est pas vraiment discuté.

16 Les réflexions consacrées à la « race » concernent la situation singulière des États-Unis, avec ses repères (plusieurs exemples sont dérivés des conséquences de la conception de la «goutte de sang») et sa crispation autour de la situation de la minorité noire. En 
effet, même si cet ouvrage mentionne les questions «raciales » chez les Amérindiens, les Asiatiques ou les Hispaniques, les termes du débat sont propres au clivage de la nation états-unienne par rapport à sa population noire (où le «métis » n'existe pas). À l'heure actuelle, on tend vers une situation médiane, moins rigide qu'il y a 50 ans (où les cases ethniques reflétaient une vision raciale étanche et permettant très peu de catégories), mais qui ne relève pas de l'« empire du choix ». Comme on l'a dit, pour des raisons liées à la stratification sociale (les populations racisées demeurent les moins favorisées socialement), « les polices de la race » restent très présentes.

17 On appréciera la clarté de l'exposé de cet ouvrage, ainsi que la documentation et les références, très riches. On peut juste regretter qu'elles soient limitées à la sphère anglo-saxonne. À cet égard, nous pensons que des auteurs comme Colette Guillaumin, qui ont pensé et théorisé l'articulation entre le sexe et la race comme modes de catégorisation et infériorisation sociale, auraient avantageusement pu être consultés.

\section{AUTEURS}

OLGA L. GONZALEZ

CNRS / Universités Paris Diderot - Nice Sophia Antipolis, URMIS 